\title{
Suboptimal management of hypertriglyceridemia in the outpatient setting is associated with the recurrent pancreatitis
}

\section{Ping Yan}

Affilated hospital of southwest medical university

Hong-Xian Zhao

Southwest medical university

xia chen ( -970217858@qq.com )

Affilated hospital of southwest medical university https://orcid.org/0000-0003-3675-5336

Research article

Keywords: acute pancreatitis; hypertriglyceridemia; recurrence; outpatient setting

Posted Date: October 10th, 2019

DOI: https://doi.org/10.21203/rs.2.15878/v1

License: (1) (i) This work is licensed under a Creative Commons Attribution 4.0 International License.

Read Full License 


\section{Abstract}

Background Hyperlipemia is a well-established etiology of acute pancreatitis (AP). However, few data are available in the medical literature about the management of triglyceride levels in the outpatient setting in patients with hypertriglyceridemic acute pancreatitis (HTG-AP). We evaluated the blood triglyceride levels and the follow-up of triglyceride management in patients with HTG-AP.

Methods This retrospective study enrolled patients with HTG-AP from January 2013 to March 2019 in Affiliated Hospital of Southwest Medical of University. By reviewing the hospitalization records and the follow-up data, the clinical features, blood triglyceride levels, lipid-lowering medications use and blood triglyceride levels monitoring after hospital discharge were analyzed.

Results 133 patients ( 46 women, 87 men; median age at presentation 37.4 years) diagnosed with HTG-AP were enrolled in the study. 32 cases $(24.1 \%)$ presented with recurrent acute pancreatitis (RAP). Patients who had RAP were younger and had higher blood triglyceride levels compared with that of single attack ( $P<0.05)$. No difference of serum amylase levels, hospitalization duration and mortality rate were observed between non-RAP and RAP. Lipid monitoring was only observed in $12.8 \%$ of patients and 10 patients $(7.5 \%)$ took medications to control blood triglyceride levels after hospital discharge. The followup of triglyceride levels in the outpatient setting were higher in RAP patients than that of non-recurrent cases $(P<0.05)$. Among the patients who had measured their triglyceride levels after discharge, $83.3 \%$ of patients with RAP had at least 1 follow-up of triglyceride level that higher than $500 \mathrm{mg} / \mathrm{dL}$, while no patient had one HTG-AP attack displayed triglyceride levels higher than $500 \mathrm{mg} / \mathrm{dL}$.

Conclusions Triglyceride levels after hospital discharge higher than $500 \mathrm{mg} / \mathrm{dL}$ may be associated with an increasing risk of relapse of clinical acute pancreatitis events. Inappropriate management of triglyceride control in the outpatient setting may be associated with an increasing risk of relapse of clinical HTG-AP events.

\section{Background}

Acute pancreatitis (AP) is now one of the most common reasons for hospitalization with a gastrointestinal condition. It is widely reported that the most common causes are excessive alcohol consumption and gallstones. Hypertriglyceridemia (HTG) is the third cause of AP in the western population [1]. The incidence of AP related to elevated triglycerides (TG) is estimated up to 10 percent of total cases [2]. But in China, HTG is more frequent as a cause of AP, ranging from $14.3 \%$ to $24 \%$ [3-6]. It has been shown that insufficient intake of vegetable and fruit, physical inactivity, and overweight or obesity were high in the floating population in China, which may be partly explain the prevalence of AP induced by HTG increased dramatically in recent years.

Hypertriglyceridemic acute pancreatitis (HTG-AP) is generally considered to have no correlation with elevated serum cholesterol levels, but to be closely associated with elevated serum TG. There are various epidemiological studies that tried to determine the appropriate cut-off for TG level to cause AP [5,7-8]. At 
least for now, HTG-AP is oftentimes established by the presence of serum TGs are $\geq 1000 \mathrm{mg} / \mathrm{dL}$. Although gallstones and alcohol are the most common etiologies for recurrent acute pancreatitis (RAP), HTG is also a well-known cause of RAP. In the management of RAP, understanding of etiology is of vital importance for establishing specific treatment and thereby decreasing the possibility of recurrence. As for HTG-AP, preventive measures such as dietary modification, antihyperlipidemic agents, lipid monitoring is indicated to be beneficial in reducing the possibility of recurrence in clinical practice. The control of TG levels can decrease the clinical pancreatitis events and associated health care costs [9].

Lipid-lowering drugs and regular monitoring of TG levels are long-term strategies in the prevention of next episode of HTG- AP, but non-compliance is frequent and many of these patients develop recurrent attacks. Few researches have focused on the long-term HTG management after HTG-AP attack has been resolved. In the present study, in addition to demographic and clinical characteristics, we mainly investigated the clinical blood TG levels and HTG management in patient who have had attack of HTG$A P$, and thus to assess the patient understanding in $H T G$ management for the prevention of pancreatitis.

\section{Methods}

We retrospectively studied all patients with HTG-AP admitted to Affiliated Hospital of Southwest Medical University (Luzhou, China) between January 2013 and March 2019. This study was reviewed and approved by the Ethics Committee of Affiliated Hospital of Southwest Medical University. AP is diagnosed as having 2 out of 3 factors: abdominal pain suggestive of AP, serum amylase and (or) lipase concentration $\geq 3$ times higher than the normal value, and abdominal imaging examination in line with imaging changes typical for AP. The diagnosis of HTG-AP is considered when serum TG levels are $>1000 \mathrm{mg} / \mathrm{dL}$ at clinical onset. RAP was defined as 2 or more well-documented separate attacks of AP with complete resolution for more than 3 months between attacks. Patients with traumatic, biliary disease, alcohol abuse, pregnancy and other causes of AP were excluded. Additionally, patients with a history of mental illness or inability to communicate were also excluded from the study.

Data recorded included the serum amylase levels at the time of hospitalization; the serum TG levels at the time of admission (the values of patients who had 2 or more disease attacks were counted as the average values of every episode); the number of total HTG-AP episode; gender; age of the first attack of HTG-AP; the length of hospital stay; mortality; history of obesity and diabetes. Lipid-lowering drugs use and blood TG levels monitoring after hospital discharge were obtained by telephone review.

The data were analyzed for significance by using SPSS 17.0. Continuous data are expressed as the mean \pm standard deviation (SD). Groups were compared using Student's $t$-test for variables with a normal distribution. Categorical data are displayed as $\mathrm{n}(\%)$ and were analyzed with the Pearson chi-square test or the Fisher exact test as appropriate. $P$-value $<0.05$ was considered significant.

\section{Results}


In total, 206 patients fulfilling the inclusion criteria of HTG-AP were considered for the study. Ultimately, 73 patients were excluded, and 133 patients were enrolled for the analysis. Of these, $101(75.9 \%)$ had one episode of HTG-AP and 32 patients (24.1\%) presented with RAP, 16 (12\%) had 2 episodes of HTG-AP, $6(4.5 \%)$ had 3 episodes, 10 (7.5\%) had 4 or more episodes. 87 patients $(65.4 \%)$ were males, and 46 $(34.6 \%)$ were females. The rate of male versus female is $1.89: 1$. The age range of the patients admitted in this study was from 15 to 75 years. The average age of patients at the first time of admission was 37.4 years, over half (55.6\%) of patients were $31-45$ years, while only $3.8 \%$ was $61-75$ years. obesity was found in 9 patients $(6.8 \%)$, diabetes in 23 patients (17.3\%). (Table 1)

We next compared the characteristics between patients with non-RAP and RAP. As it was shown in table 2 , the mean age in patients who had only one episode of HTG-AP was 43.18 7 .44 years, whereas patients with RAP were younger $(P<0.05)$, the mean age was $35.26 \pm 6.34$ years. Patients who had 4 or more episodes were significantly younger, which was $28.87 \pm 3.63$ years, $P<0.05$ (Fig. 1 ). No difference of serum amylase levels and hospitalization duration were observed between patients with single attack and recurrent attack. The mortality rate in one episode of HTG-AP was $4.2 \%$, while it was $6.3 \%$ in recurrent cases, but the difference was not statistically significant. (Table 2)

For the blood TG levels at admission, the average value was higher in patients with RAP compared to that of non-recurrent cases $(1736 \pm 273 \mathrm{mg} / \mathrm{dL}$ compared to $1279 \pm 205 \mathrm{mg} / \mathrm{dL}, P<0.05)$. The serum TG levels in patients who had 4 or more disease attacks were much higher, the average value was $2213 \pm 396$ $\mathrm{mg} / \mathrm{dL}, P<0.05$ (Fig.2). It is likely that the higher the serum TG level was, the more chance of recurrence of HTG-AP.

To our surprise, lipid-lowering drugs were only taken in 10 patients (7.5\%) after discharge, 3 in patient with single attack of HTG-AP, 7 in patients with RAP. There were 17 patients $(12.8 \%)$ observed in this study had measured their blood HTG levels after discharge, 5 in patients had non-RAP, 12 in RAP. The highest level of TG was $1578 \mathrm{mg} / \mathrm{dL}$, while the lowest was $94.7 \mathrm{mg} / \mathrm{dL}$. However, the TG levels in the outpatient setting were higher in patients with RAP than that of non-recurrent cases $(453 \pm 57 \mathrm{vs.} 229 \pm 34$ $\mathrm{mg} / \mathrm{dL}, P<0.05)$. Among the patients who had measured their TG levels after discharge, $83.3 \%$ of patients with HTG-RAP had at least 1 follow-up triglyceride laboratory result that higher than $500 \mathrm{mg} / \mathrm{dL}$, while there was no patient who had a single attack displayed TG levels higher than $500 \mathrm{mg} / \mathrm{dL}$ during follow-up.

\section{Discussion}

AP is an inflammatory disorder of the pancreas characterized by rapid onset and has multiple possible etiologies. The association between severe HTG and AP has long been recognized [10]. Yet the exact threshold of serum TG level to trigger AP has not been defined. AP is conventionally thought to be triggered when TG levels exceed 1,000 mg/ dL $(11.3 \mu \mathrm{mol} / \mathrm{L})$. It was also proposed that the TG level > $500 \mathrm{mg} / \mathrm{dL}$ should raise a high-degree suspicion of HTG-AP, especially in the absence of other probable etiologies [5]. The incidence of HTG-AP was increasing year by year especially in China [11-12]. Moreover, 
HTG-AP was reported to increase at a faster rate than alcoholic AP [6]. In some regions, HTG had exceeded alcohol and become the second cause of AP [3,13].

Clinical presentation of HTG-AP is similar to that of pancreatitis of other etiologies [14], but patients with HTG-AP were more likely to have pancreatic necrosis and organ failure, severe acute pancreatitis, and systemic inflammatory response Syndrome $[4,11]$. Compared to other causes of AP, HTG-AP patients were younger, had more hospital stays and higher recurrence rate in previous study [4]. A multicenter study showed that alcoholic pancreatitis and HTG-AP were mainly distributed in patients before the age of 49 years [6]. In the present study, half (55.5\%) of patients were 31-45 years and HTG-AP appeared to be more prevalent in males than females, which is similar to other studies, but the exact reason is not clear. HTG is common in patients with the metabolic syndrome, type 2 diabetes and obesity which can contribute to elevated TG levels substantial enough to provoke pancreatitis [15]. In the present study, the prevalence of obesity and diabetes was lower than other studies, obesity was found only in $6.8 \%$ of patients, diabetes in $17.3 \%$ of patients.

RAP is a syndrome of multiple distinct acute inflammatory responses originating within the pancreas in individuals with genetic, environmental, traumatic, morphologic, metabolic, biologic, and/or other risk factors who experienced 2 or more episodes of documented AP [16]. If unchecked, recurrent episodes of AP may lead to chronic pancreatitis. Approximately $9 \%$ to $31 \%$ of patients with AP develop recurrent attacks of pancreatitis [1,17-18]. The etiological factors of RAP are similar to those of first attack AP. Recently HTG is accepted as a major cause of RAP particularly in China. Deng et al reported that HTG accounted for $21 \%$ of RAP and biliary factor was still the leading causes of RAP in a region of China [19]. A multicenter study showed the recurrence rate of HTG-AP was obviously higher than other causes (15.29\%, 7.73\% and 9.75\% for HTG-AP, biliary AP and alcoholic AP respectively) [6]. The present study showed that $24.1 \%$ of patients with HTG-AP develop a recurrent disease. Patients with RAP seemed younger than non-recurrent cases. In contrast, serum amylase levels, hospitalization duration and mortality rate were almost identical in the two groups. Patients with RAP in the present study presented with higher blood TG levels at the time of admission, suggesting that blood TG level is likely associated with the relapse of HTG-AP.

Potential causes should be carefully evaluated in patients with an initial episode of AP. If the underlying cause is not corrected, any factor responsible for pancreatitis can lead to recurrent episodes. At present, there is a lack of consensus on the most appropriate treatment options for patients with HTG-AP. Although there are conflicting opinions regarding the lipid-lowering therapy for asymptomatic HTG to prevent AP [20-21]. However, when TG levels are $\geqq 500 \mathrm{mg} / \mathrm{dL}$, and especially when they are $\geqq 1000$ $\mathrm{mg} / \mathrm{dL}$, the primary treatment strategy is to reduce TG levels with a TG-lowering drug to reduce the risk of pancreatitis [22]. For those who had suffered HTG-AP, diet control, exercise program, lipid-lowering agents and plasma exchange are recommended to reduce TG levels during the acute phase of HTG-AP, and in the prevention of recurrence. In present study, the follow-up of blood TG levels after hospital discharge were investigated. We found that the TG levels were higher in patients with RAP than that of non-RAP. Among the patients who had measured TG levels after discharge, the majority (83.3\% \of RAP had at least 
1 follow-up of TG laboratory result higher than $500 \mathrm{mg} / \mathrm{dL}$, while there was no patient who had one episode displayed TG levels higher than $500 \mathrm{mg} / \mathrm{dL}$. The risk of AP increases in a direct relationship to the level of TG [23]. In a retrospective cohort study, the risk of incident AP increased by $4 \%$ for every $100-$ $\mathrm{mg} / \mathrm{dL}$ increase in TG concentration [8]. Lower follow-up of TG levels was associated with a lower incidence of important clinical events for patients with severe HTG [24]. It is generally accepted that it is important to treat HTG to minimize the risk of recurrence of AP by reducing TG levels to $<500 \mathrm{mg} / \mathrm{dL}$ $[15,25]$. Our results also supported that follow-up of TG levels were $<500 \mathrm{mg} / \mathrm{dL}$ have less chance of pancreatitis episode and may be helpful in reducing the recurrence of pancreatitis.

Once the HTG-AP attack has been resolved, prevention of a next episode is compulsory. The long-term management consists of dietary intervention, long-term medications, regular monitoring of serum TG levels and lifestyle modifications [15]. In the present study, there were only $7.5 \%$ of patients continued to use the lipid-lowering drugs after hospital discharge. The unsatisfied medication compliance is likely an important contributing factor to the high frequency of RAP in our study. Lipid monitoring might improve control of lipid parameters and the dietary compliance of patients with HTG [26]. However, the percentage of follow-up of TG level monitoring was relatively low in our study, only $12.8 \%$ of patients had their TG levels measured after discharge, showing that majority of the patients in present study lacked the understanding of HTG control for the prevention of AP attack. Although strategies of community based chronic disease treatment have been established in China, identifying and resolving compliance issues of HTG management should be improved and valued in these HTG-AP patients as well as decrease the recurrence rate.

\section{Conclusions}

In summary, this retrospective study found that patients with RAP seemed younger and had higher blood TG levels. TG levels are $\geqq 500 \mathrm{mg} / \mathrm{dL}$ in the outpatient setting may be associated with an increasing risk of relapse of clinical HTG-AP events. The majority of patients in the present study failed to routinely TG levels monitoring and serum TG control, this inappropriate management of TG control partly contributed to the high recurrence rate in the present study. Therefore, importance of HTG management in the outpatient setting should be attached in the long-term treatment of severe HTG and the prevention of next episode of HTG-AP.

\section{List Of Abbreviations}

AP - acute pancreatitis

HTG - hypertriglyceridemia

HTG-AP - hypertriglyceridemic acute pancreatitis

RAP - recurrent acute pancreatitis 


\section{Declarations}

Acknowledgements $\square$ Not Applicable.

Authors' contributions: Ping-yan did the work of clinical investigation. Hong-xian Zhao analyzed and interpreted the patient data. Xia Chen designed the research and was a major contributor in writing the manuscript. All authors read and approved the final manuscript.

Funding: No funding was obtained for the study.

Availability of data and materials: Not applicable.

Ethics approval and consent to participate: Not applicable.

Consent for publication: Not Applicable.

Competing interests: The authors declare no competing interests.

\section{References}

1. Gullo L, Migliori M, Pezzilli R, Oláh A, Farkas G, Levy P, et al. An update on recurrent acute pancreatitis: data from five European countries. Am J Gastroenterol. 2002;97:1959-1962. doi: 10.1111/j.1572-0241.2002.05907.x.

2. Valdivielso P, Ramirez-Bueno A, Ewald N. Current knowledge of hypertriglyceridemic pancreatitis. Eur J Intern Med. 2014;25:689-694. doi: 10.1016/j.ejim.2014.08.008.

3. Zhu Y, Pan X, Zeng H, He W, Xia L, Liu P, et al. A Study on the Etiology, Severity, and Mortality of 3260 Patients With Acute Pancreatitis According to the Revised Atlanta Classification in Jiangxi, China Over an 8-Year Period. Pancreas. 2017; 46:504-509. doi:10.1097/MPA.0000000000000776.

4. Qiu L, Sun RQ, Jia RR, Ma XY, Cheng L, et al. Comparison of Existing Clinical Scoring Systems in Predicting Severity and Prognoses of Hyperlipidemic Acute Pancreatitis in Chinese Patients: A Retrospective Study. Medicine (Baltimore). 2015;94:e957. doi: 10.1097/MD.0000000000000957.

5. Zhang XL, Li F, Zhen YM, Li A, Li A, Fang Y. Clinical Study of 224 Patients with Hypertriglyceridemia Pancreatitis. Chin Med J (Engl). 2015;128:2045-2049. doi:10.4103/0366-6999.161361

6. Zheng Y, Zhou Z, Li H, Li J, Li A, et al. A multicenter study on etiology of acute pancreatitis in Beijing during 5 years. Pancreas. 2015; 44:409-414. doi:10.1097/MPA.0000000000000273.

7. Sandhu S, Al-Sarraf A, Taraboanta C, Frohlich J, Francis GA. Incidence of pancreatitis, secondary causes, and treatment of patients referred to a specialty lipid clinic with severe hypertriglyceridemia: a retrospective cohort study. Lipids Health Dis. 2011;10:157. doi: 10.1186/1476-511X-10-157. 
8. Murphy MJ, Sheng X, MacDonald TM, Wei L. Hypertriglyceridemia and acute pancreatitis. JAMA Intern Med. 2013;173:162-164. doi: 10.1001/2013.jamainternmed.477.

9. Vipperla K, Somerville C, Furlan A, , Koutroumpakis E, Saul M, et al. Clinical Profile and Natural Course in a Large Cohort of Patients With Hypertriglyceridemia and Pancreatitis. J Clin Gastroenterol. 2017;51:77-85. doi:10.1097/MCG.0000000000000579.

10. Toth PP, Grabner M, Ramey N, Higuchi K. Clinical and economic outcomes in a real-world population of patients with elevated triglyceride levels. Atherosclerosis. 2014;237:790-797. doi: 10.1016/j.atherosclerosis.2014.09.029.

11. Huang YX, Jia L, Jiang SM, Wang SB, Li MX, Yang BH. Incidence and clinical features of hyperlipidemic acute pancreatitis from Guangdong, China: a retrospective multicenter study. Pancreas. 2014;43:548-552. doi: 10.1097/MPA.0000000000000069.

12. Yin G, Cang X, Yu G, Hu G, Ni J. Different Clinical Presentations of Hyperlipidemic Acute Pancreatitis: A Retrospective Study. Pancreas. 2015; 44: 1105-1110. doi: 10.1097/MPA.0000000000000403.

13. Fan J, Ding L, Lu Y, Zheng J, Zeng Y, Huang C. Epidemiology and Etiology of Acute Pancreatitis in Urban and Suburban Areas in Shanghai: A Retrospective Study. Gastroenterol Res Pract. 2018;2018:1420590. doi: 10.1155/2018/1420590.

14. Scherer J, Singh VP, Pitchumoni CS, Yadav D. Issues in hypertriglyceridemic pancreatitis: an update. J Clin Gastroenterol. Mar 2014;48:195-203. doi: 10.1097/01.mcg.0000436438.60145.5a.

15. Lindberg DA. Acute pancreatitis and hypertriglyceridemia. Gastroenterol Nurs. 2009;32:75-82; quiz 83-74. doi: 10.1097/SGA.0b013e31819de3e0.

16. Guda NM, et al. Recurrent Acute Pancreatitis: International State-of-the-Science Conference With Recommendations. Pancreas. 2018;47:653-666. doi: 10.1097/MPA.0000000000001053.

17. Zhang W, Shan HC, Gu Y. Recurrent acute pancreatitis and its relative factors. World J Gastroenterol. 2005;11:3002-3004. doi: :10.3748/wjg.v11.i19.3002.

18. Gao YJ, et al. Analysis of the clinical features of recurrent acute pancreatitis in China. $J$ Gastroenterol. 2006;41:681-685. doi:10.1007/s00535-006-1820-3.

19. Deng YY, Wang R, Wu H, Tang CW, Chen XZ. Etiology, clinical features and management of acute recurrent pancreatitis. J Dig Dis. 2014;15(10):570-577. doi: 10.1111/1751-2980.12180.

20. Lederle FA, Bloomfield HE. Hypertriglyceridemia and Pancreatitis-New Evidence That Less Is More. JAMA Intern Med. 2017;177:744-745. doi: 10.1001/jamainternmed.2017.0602.

21. Pedersen SB, Langsted A, Nordestgaard BG. Hypertriglyceridemia and Pancreatitis-New Evidence That Less Is More-Reply. JAMA Intern Med. 2017; 177: 745-746. doi: 10.1001/jamainternmed.2017.0616.

22. Karalis DG. A Review of Clinical Practice Guidelines for the Management of Hypertriglyceridemia: A Focus on High Dose Omega-3 Fatty Acids. Adv Ther. 2017; 34: 300-323. doi: 10.1007/s12325-0160462-y. 
23. Wu BU, Batech M, Dong EY, Duan L, Yadav D, Chen W. Influence of Ambulatory Triglyceride Levels on Risk of Recurrence in Patients with Hypertriglyceridemic Pancreatitis. Dig Dis Sci. 2019;64:890-897. doi: 10.1007/s10620-018-5226-x.

24. Christian JB, Arondekar B, Buysman EK Jacobson TA, Snipes RG, Horwitz R. Determining triglyceride reductions needed for clinical impact in severe hypertriglyceridemia. Am J Med. 2014;127:36-44 e31. doi: 10.1016/j.amjmed.2013.09.018.

25. Christian JB, Arondekar B, Buysman EK, Johnson SL, Seeger JD, Jacobson TA. Clinical and economic benefits observed when follow-up triglyceride levels are less than $500 \mathrm{mg} / \mathrm{dL}$ in patients with severe hypertriglyceridemia. J Clin Lipidol. 2012;6:450-461. doi: 10.1016/j.jacl.2012.08.007.

26. Hauenschild A, Ewald N, Schnell-Kretschmer H, Porsch-Oezcueruemez M, Kloer HU, et al. Successful long-term treatment of severe hypertriglyceridemia by feedback control with lipid self-monitoring. Ann Nutr Metab. 2008;52:215-220. doi: 10.1159/000138126.

\section{Tables}

Table 1 Clinical features of HTG-AP patients

\begin{tabular}{ccc}
\hline & Number & $\%$ \\
\hline Gender & & \\
Male & 87 & 65.4 \\
\hline Female & 46 & 34.6 \\
\hline Age(years) & & \\
\hline $15-30$ & 10 & 7.5 \\
\hline $31-45$ & 74 & 55.6 \\
\hline $46-60$ & 44 & 33 \\
\hline $61-75$ & 5 & 3.8 \\
\hline Obesity & 9 & 6.8 \\
\hline Diabetes & 23 & 17.3 \\
\hline
\end{tabular}

Table 2 Clinical features of non-RAP and RAP cases 


\begin{tabular}{llll}
\hline & Non-RAP & RAP & $P$ value \\
\hline Mean age (years) & $43.18 \pm 7.44$ & $35.26 \pm 6.34$ & 0.046 \\
Male n(\%) & $62(46.6 \%)$ & $25(18.8 \%)$ & \\
\hline Female n(\%) & $39(29.3 \%)$ & $7(5.5 \%)$ & \\
\hline Serum amylase levels (U/L) & $649.55 \pm 27.37$ & $714.59 \pm 28.53$ & NS \\
\hline Hospitalization duration (days) & $8.05 \pm 3.85$ & $7.39 \pm 4.28$ & NS \\
\hline Mortality n(\%) & $4(4.0 \%)$ & $2(6.3 \%)$ & NS \\
\hline TG levels (mg/dL) & $1279 \pm 205$ & $1736 \pm 273$ & 0.013 \\
\hline
\end{tabular}

NS: not significant

$P$ values $<0.05$ are considered statistically significant

Figures 


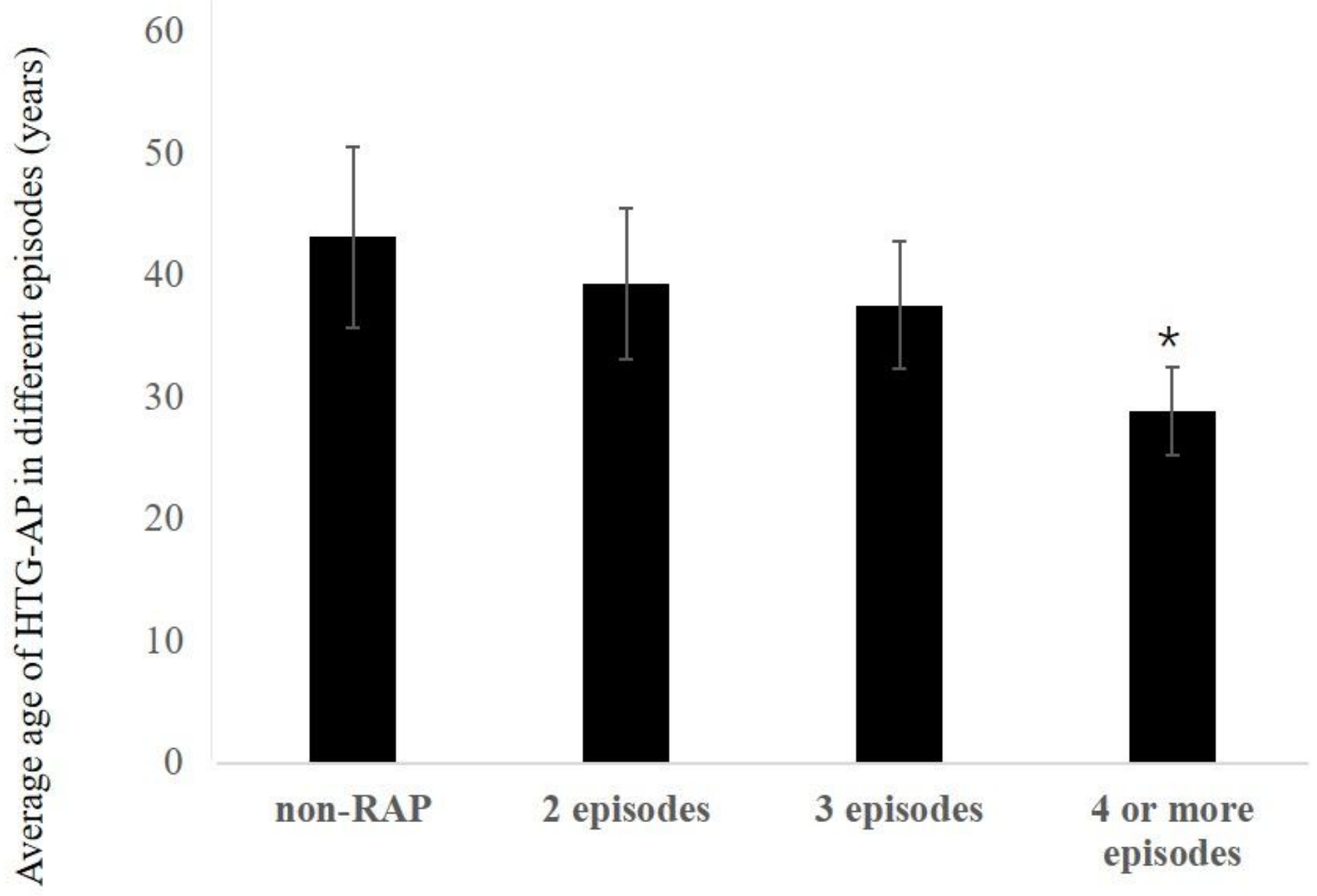

Figure 1

Average age of Patients with HTG-AP. The mean age in patients who had one episode of HTG-AP was $43.18 \pm 7$ years, patients had 4 or more episodes was $28.87 \pm 3.63$ years, which was significantly younger. * $\mathrm{P}<0.05$ denotes a significant difference compare with patients with non-RAP. 


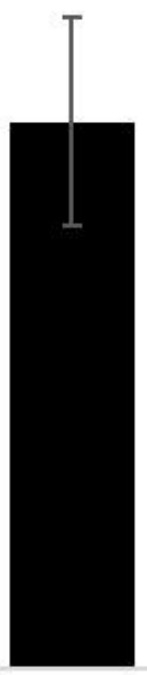
non-RAP
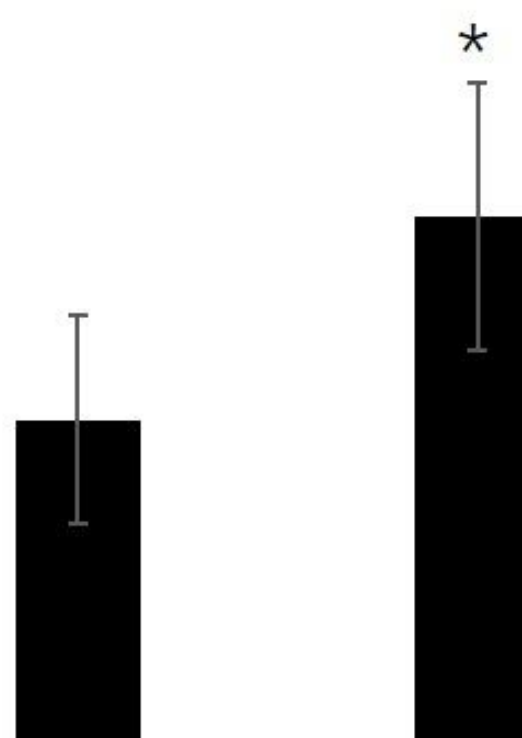